\title{
Repercusión del optimismo y de los Cinco Grandes factores de la personalidad sobre la salud de personas mayores*
}

\author{
Impact of Optimism and the "Big Five" Personality \\ Factors on Older Persons' Health
}

Recibido: julio 26 de 2011 | Revisado: junio 272013 | Aceptado: marzo 27 de 2014

\author{
José JESÚS GÁZQUEZ LINARES ** \\ María del CARMen Pérez Fuentes *** \\ ISABEL MERCADER RUBIO ***** \\ Universidad de Almería, España \\ CÁNDIDO José Inglés SAURA ****** \\ Universidad Miguel Hernández de Elche, España
}

doi:10.11144/Javeriana.UPSY13-3.rocg

Para citar este artículo: Gázquez, J. J., Pérez, M. del C., Mercader, I. \& Inglés, C. J. (2014). Repercusión del optimismo y de los Cinco Grandes factores de personalidad sobre la salud de personas mayores. Universitas Psychologica, 13(3), 995-1004. http:/ dx.doi.org/10.11144/Javeriana.UPSY13-3.rocg

* Artículo de investigación

** Director del Departamento de Psicología Evolutiva y de la Educación. Correo electrónico: jlinares@ual.es

**** Secretaria del Departamento de Psicología Evolutiva y de la Educación. Correo electrónico: mpf421@ual.es

****** Decana de la Facultad de Ciencias de la Educación, Enfermería y Fisioterapia. Profesora Contratada. Correo electrónico: imercade@ual.es

******** Profesor Titular de Universidad. Departamento de Psicología de la Salud. Correo electrónico: cjingles@umh.es

\section{RESUMEN}

Este trabajo analiza la influencia del Optimismo Disposicional y los Cinco Grandes factores de la personalidad en la percepción de salud de los mayores, así como el análisis que optimistas y pesimistas realizan respecto a su salud. La muestra está formada de 498 personas mayores $(M=63.3$ años y $D E$ $=8.55)$. Los resultados ponen de manifiesto que las personas optimistas muestran mejor percepción de su salud y menores niveles de Disfunción Social y Depresión. El factor Neuroticismo influye en los Síntomas Somáticos, Ansiedad, Disfunción Social y Depresión y es un factor clave en la relación que se establece entre el Optimismo Disposicional y la subescala de Depresión. Por otra parte, el Optimismo, solo influye directamente en la subescala Disfunción Social.

Palabras clave

optimismo; personalidad; percepción de salud; mayores

\footnotetext{
A B S T R A C T

This work studies the influence of Dispositional Optimism and the "Big Five" personality factors on older peoples' self-rated health and it examines optimists' and pessimists' analyses of their own health. The sample is made up of 498 older people $(M=63.3$ years and $S D=8.55)$. The results reveal that optimists have a better perception of their health and lower levels of social dysfunction and depression. The Neuroticism factor affects to the Somatic Symptoms, Anxiety, Social Dysfunction, and Depression; and is a key factor in the relation established between Dispositional Optimism and the subscale of Depression. However, Optimism only directly affects the subscale Social Dysfunction.

Keywords

optimism; personality; self-rated health; older people
} 
José Jesús GázQuez Linares, María del Carmen Pérez Fuentes, Isabel Mercader Rubio, CÁndido José Inglés Saura

\section{Introducción}

Son múltiples las investigaciones que analizan la relación entre la personalidad y la salud, en concreto, los Cinco Grandes factores y su influencia en esta (Friedman \& Rosenman, 1959; GómezAngulo \& Campo-Arias, 2011). En la actualidad, el interés de los autores se centra en el optimismo y su relación con la salud, habiendo surgido en los últimos años varios metaanálisis, así como un elenco amplio de investigaciones (Carver, Scheier \& Segerstrom, 2010; Londoño, Hernández, Alejo \& Pulido, 2013; Rasmussen, Scheier \& Greenhouse, 2009). La personalidad se asocia con el comportamiento de salud, pero en esta relación también interviene el optimismo que podría verse afectado, es decir, la personalidad puede influir en el optimismo y este a su vez en el comportamiento de salud (Ingledew \& Brunning, 1999). Entre los estudios que relacionan optimismo y salud se debe diferenciar entre los que analizan la influencia del optimismo en los comportamientos de salud (Lauriola, Russo, Lucidi, Violani \& Levin, 2005; Meliá \& Becerril, 2009; Radcliffe \& Klein, 2002; Vollrath \& Torgersen, 2008), en la percepción de la salud (Rieffe, Villanueva, Adrián \& Górriz, 2009) y aquellos que los hacen en la salud real (Booth-Kewley \& Friedman, 1987).

El factor Optimismo ha sido ampliamente estudiado aunque su estabilidad en el tiempo es discutida, encontrando algunos estudios una elevada correlación (Atienza, Stephens \& Townsend, 2004), mientras que otros indican cambios en los niveles de optimismo ante las diferentes situaciones a las que el sujeto debe enfrentarse (Segerstrom, 2007; Sweeny, Carroll \& Shepperd, 2006). Optimismo y pesimismo son definidos como expectativas o creencias generalizadas positivas y/o negativas que el sujeto tiene acerca de su futuro (Scheier \& Carver, 1985, 1987). La heredabilidad del optimismo se fija en el 25\% (Plomin et al., 1992), porcentaje más bajo que otros componentes de la personalidad sobre el que también influyen otros factores, tales como: el ambiente familiar durante la infancia (Heinonen, Räikkönen \& Keltikangas-Järvinen, 2005), el estatus socioeconómico (Heinonen et al., 2006), la realización de actividades sociales y educativas (Gázquez, Pérez \& Carrion, 2010; de la Cruz-Sánchez, Feu \& Vizuete-Carrizosa, 2013) y el Neuroticismo (Chico, 2002; Darvill \& Johnson, 1991; Scheier, Carver \& Bridges, 1996). En esta etapa de la vida, donde se encuentran inmersas las personas mayores, es decir en la vejez, no se caracteriza por el sufrimiento emocional (Charles, Reynolds \& Gatz, 2001), aunque si se aprecia una menor intensidad de las emociones positivas y un mayor nivel de afecto negativo que en etapas evolutivas anteriores (Márquez, Fernández, Montorio \& Losada, 2008).

El Optimismo se asocia negativamente con la presencia de síntomas de malestar físico Scheier y Carver (1985), predice una menor incidencia de síntomas depresivos en el sujeto (Giltay, Zitman \& Kromhout, 2006), existiendo también una relación negativa entre ambos (Shnek, Irvine, Stewart \& Abbey, 2001). Otros factores de la personalidad también han sido estudiados en relación con la salud, como el Neuroticismo, que se relaciona positivamente con el número de síntomas médicos (Costa \& McCrae, 1985; Zanon \& Hutz, 2013), aunque ese factor psicosocial puede estar contaminando la medida subjetiva de salud (Rasmussen et al., 2009), puesto que en algunos casos ha sido asociado directamente con el relato de síntomas infundados (Feldman, Cohen, Doyle, Skoner \& Gwaltney, 1999).

El análisis de los Cinco Grandes factores de la personalidad en relación con la percepción de la salud nos muestra una correlación significativa y negativa respecto a: Estabilidad Emocional, Extraversión y Responsabilidad (Johnson, Batey \& Holdsworth, 2009). Debido a todo ello, a través de este estudio se pretende conocer la validez predictiva del Optimismo Disposicional (OpD) y los Cinco Grandes (Neuroticismo, Extraversión, Amabilidad, Apertura y Responsabilidad), respecto a la percepción de salud de los mayores.

\section{Método}

\section{Participantes}

Los sujetos se seleccionaron al azar, atendiendo a las normas éticas y siendo respetuosos con la confidencialidad (Cabanach, Fariña, Freire, González 
\& Ferradás, 2013; Del Barrio et al., 2011; Oyanadel \& Buela-Casal, 2011; Inglés, Martínez-González \& García-Fernández, 2013; Torregrosa, Inglés, GarcíaFernández, Valle \& Núñez, 2012; Rodríguez et al., 2011; Vizcarra \& Pòo, 2011) de todos los participantes de la muestra de mayores de 60 años que residían en los diferentes Centros de Día y Residencias para Mayores de la provincia de Almería. La muestra estuvo compuesta por un total de 498 participantes con edades comprendidas entre los 60 y 90 años $(\mathrm{M}=$ 63.9 de edad y $D E=8.55)$, de los cuales un $45.6 \%(\mathrm{~N}$ $=227)$ eran hombres, con edad media de 64.6 años $(D E=8.96)$, y el resto de la muestra, el $54.4 \%(N$ $=271$ ) eran mujeres, con edad media de 63.31 años $(D E=8.205)$. En función del estado civil, el 71.3\% de la muestra estaba casado/a, el 16.9\% viudo/a, el $6.7 \%$ separado/a o divorciado/a y el $5.1 \%$ era soltero/a.

\section{Instrumentos}

Para la consecución de objetivo de la investigación se han empleado los siguientes instrumentos de medida.

Cuestionario NEO-FFI (Costa 8 McCrae, 1999)

Una versión reducida que recoge 60 elementos para la evaluación de la personalidad según el modelo de los Cinco Grandes (Neuroticismo, Extraversión, Apertura, Amabilidad y Responsabilidad).

Test de Orientación Vital Revisado ([LOT-R]; Sheier, Carver $\mathbb{E}$ Bridges, 1994)

En su adaptación española (Otero, Luengo, Romero, Gómez \& Castro, 1998) que consta de 10 ítems en escala Likert de 5 puntos, de los cuales seis miden la dimensión de Optimismo Disposicional (OpD) (tres redactados en sentido positivo y tres en sentido negativo), mientras que los otros cuatro restantes son de relleno y sirven para hacer menos evidente el contenido del test.

Cuestionario de Salud General ([GHQ28]; Goldberg 83 Hillier, 1979)

Este cuestionario, centrado fundamentalmente en los componentes psicológicos de la salud, está destinado a detectar trastornos psíquicos. Analiza dos fenómenos: la posibilidad por parte del sujeto de desarrollar actividades normales y la aparición de nuevas dolencias psíquicas. Posee cuatro componentes con siete ítems cada uno: síntomas somáticos, ansiedad e insomnio, disfunción social y depresión grave.

Test del Dibujo del Reloj ([TDR]; Cacho, GarcíaGarcía, Arcaya, Vicente $\mathcal{E}$ Lantada, 1999)

Prueba de detección (screening) para valorar el estado cognitivo del sujeto. Evalúa los distintos mecanismos implicados en la realización de tareas, como funciones visoperceptivas, visomotoras y visoconstructivas, planificación y ejecución motoras.

\section{Procedimiento}

Una vez solicitado y obtenido el consentimiento, tanto de los responsables de los centros como de los propios participantes, se procede a la obtención de los datos, para lo cual se instruyó a cuatro personas que fueron las encargadas de aplicar los diferentes cuestionarios a la muestra que participó en el estudio. Una vez se llevó a cabo la implementación de las correspondientes pruebas y se realizó su corrección, se procedió a la elaboración e introducción de los datos en la respectiva base de datos y se procedió a su análisis a través del programa estadístico SPSS 15.0. Antes de proceder a la introducción de los datos, se debe indicar que el Test del Dibujo del Reloj (TDR) (Cacho et al., 1999) fue aplicado a toda la muestra con el objeto de detectar de forma sencilla y rápida un posible deterioro cognitivo, por lo que aquellos sujetos que en este test mostraban indicios de deterioro, fueron eliminados de la muestra, en concreto dos.

\section{Análisis de datos}

Se realizaron correlaciones bivariadas para conocer la relación entre las diferentes variables analizadas y parciales, con el objetivo de controlar la influencia de las diferentes variables de la personalidad sobre la personalidad en general y las cuatro escalas de 
salud del GHQ-28. Por otro lado, para conocer si existen diferencias en el nivel de salud de las personas optimistas y pesimistas, se dividió la muestra en dos, utilizando para ello el cálculo de la mediana o el percentil 50 cuyo valor en la prueba LOT-R va a dejar el mismo número de casos antes y después de él, procedimiento ya utilizado con esta prueba (Martínez, Reyes, García \& González, 2006). Siendo la puntuación 17, el punto de corte tomado para la diferenciación de ambos grupos, según la puntuación fuera menor/igual (pesimismo disposicional $=$ puntuaciones entre 6 y 17) o superior $(\mathrm{OpD}=$ puntuaciones entre 18 y 25). La comparación entre ambos grupos fue efectuada a través de la prueba $t$ para muestras independientes. La construcción del modelo explicativo de la salud percibida, se ha llevado a cabo a través del análisis de regresión, utilizando el método de pasos sucesivos.

\section{Resultados}

En el análisis de la relación entre las diferentes variables del estudio (Tabla 1) podemos destacar que el factor Neuroticismo muestra una correla- ción positiva y significativa con la puntuación total del cuestionario de salud, así como con el resto de las subescalas, mostrando altas puntuaciones. Esta relación nos indica que cuanto mayor son las puntuaciones en Neuroticismo, también lo son las puntuaciones en las diferentes variables de la salud, es decir, a mayores niveles de Neuroticismo peor percepción de la salud. Con índices un poco inferiores, pero igualmente mostrando una correlación significativa, aunque en este caso negativa, se encuentra el factor Extraversión. Bajas puntuaciones en Extraversión se relacionan con altas puntuaciones en las variables de la salud, es decir, a menores puntuaciones en Extraversión peor percepción de la salud.

El análisis de las relaciones entre el $\mathrm{OpD}$ y los Cinco Grandes indica que las correlaciones son positivas y significativas respecto a la Extraversión $(r$ $=0.32)$, la Amabilidad $(r=0.24)$, la Apertura $(r=$ 0.36 ) y la Responsabilidad $(r=0.2)$ e igualmente significativas pero negativas, en el Neuroticismo $(r=-0.32)$.

Se observa como la correlación entre el OpD y la salud es significativa y negativa, tanto respecto

TABLA 1

Correlaciones de Pearson entre todas las variables utilizadas en el estudio

\begin{tabular}{|c|c|c|c|c|c|c|c|c|c|c|c|}
\hline & $\begin{array}{c}\text { GHQ } \\
\text { TOTAL }\end{array}$ & GHQ A & GHQ B & GHQ C & GHQD & NEO N & NEOE & NEO O & NEO A & NEOC & LOT-R \\
\hline $\begin{array}{l}\text { GHQ } \\
\text { TOTAL }\end{array}$ & 1 & $0.759 * *$ & $0.748 * *$ & $0.68 * *$ & $0.779 * *$ & $0.566^{* *}$ & $-0.235 * *$ & -0.025 & $-0.181^{*}$ & $-0.195 * *$ & $-0.261 * *$ \\
\hline GHQ A & & 1 & $0.445 * *$ & $0.425^{* *}$ & $0.403^{* *}$ & $0.438 * *$ & $-0.195 * *$ & -0.139 & $-0.169^{*}$ & -0.130 & $-0.175^{*}$ \\
\hline GHQ B & & & 1 & $0.323 * *$ & $0.532 * *$ & $0.468^{* *}$ & $-0.246 * *$ & -0.037 & -0.094 & 0.065 & $-0.179 *$ \\
\hline GHQ C & & & & 1 & $0.537^{* * *}$ & $0.336^{* * *}$ & $-0.24 * *$ & -0.137 & $-0.227^{* *}$ & $-0.301 * *$ & $-0.326^{* *}$ \\
\hline GHQ D & & & & & 1 & $0.46 * *$ & $-0.167^{*}$ & 0.119 & -0.057 & $-0.17^{*}$ & $-0.189^{* * *}$ \\
\hline NEO N & & & & & & 1 & $-0.326^{* *}$ & -0.101 & $-0.318^{* *}$ & $-0.236^{* *}$ & $-0.321^{* *}$ \\
\hline NEO E & & & & & & & 1 & $0.349 * *$ & $0.338 * *$ & 0.138 & $0.32 * *$ \\
\hline NEO O & & & & & & & & 1 & 0.059 & $0.279 * *$ & $0.243 * *$ \\
\hline NEO A & & & & & & & & & 1 & $0.237 * *$ & $0.364 * *$ \\
\hline NEO C & & & & & & & & & & 1 & $0.205 * *$ \\
\hline LOT-R & & & & & & & & & & & 1 \\
\hline
\end{tabular}

Nota. LOT-R (Puntuación Total del Test de Orientación Vital-Revisado); NEO N (Factor Neuroticismo); NEO E (Factor Extraversión); NEO O (Factor Amabilidad); NEO A (Factor Apertura); NEO C (Factor Responsabilidad); GHQ TOTAL (Puntuación Total del Cuestionario de Salud General de Goldberg ); GHQ A (Factor Síntomas Somáticos del Cuestionario de Salud General de Goldberg); GHQ B (Factor Ansiedad del Cuestionario de Salud General de Goldberg); GHQ C (Factor Disfunción Social del Cuestionario de Salud General de Goldberg); GHQ D (Factor Depresión del Cuestionario de Salud General de Goldberg).

** La correlación es significativa al nivel 0.01 (bilateral).

* La correlación es significativa al nivel 0.05 (bilateral).

Fuente: elaboración propia 
RePercusión del optimismo y de los Cinco GRANDES FACTORES DE LA

PERSONALIDAD SOBRE LA SALUD DE PERSONAS MAYORES

Tabla 2

Correlaciones bivariadas y parciales (controlando el efecto de los Cinco Grandes) del optimismo disposicional con la salud general y las cuatro escalas de salud

\begin{tabular}{cccccc}
\hline & GHQ TOTAL & GHQ A & GHQ B & GHQ C & GHQ D \\
\hline LOT-R & $-0.26^{* *}$ & $-0.17^{*}$ & $-0.18^{*}$ & $-0.33^{* *}$ & $-0.19^{* *}$ \\
NEO N & -0.1 & -0.04 & -0.03 & $-0.25^{* *}$ & -0.05 \\
NEO E & $-0.2^{* *}$ & -0.12 & -0.11 & $-0.27^{* *}$ & $-0.14^{*}$ \\
NEO O & $-0.26^{* *}$ & $-0.15^{*}$ & $-0.18^{*}$ & $-0.31^{* *}$ & $-0.23^{*}$ \\
NEO A & $-0.21^{* *}$ & -0.12 & $-0.16^{*}$ & $-0.27^{* *}$ & $-0.18^{*}$ \\
NEO C & $-0.23^{* *}$ & $-0.15^{*}$ & $-0.20^{* *}$ & $-0.29^{* *}$ & $-0.16^{*}$ \\
\hline
\end{tabular}

Nota. Se han utilizado las mismas abreviaturas que en la Tabla 1.

$* * \mathrm{p}<0.01$ (bilateral).

$* \mathrm{p}<0.05$ (bilateral).

Fuente: elaboración propia

a la puntuación total de salud como en las cuatro subescalas. Así, las altas puntuaciones en OpD (optimismo) estarían relacionadas con menores y, por tanto, mejores puntuaciones en la autopercepción de la salud por parte de los mayores, no solo respecto a la puntuación total de salud percibida, sino al resto de los aspectos analizados por las subescalas.

Respecto a la relación entre el $\mathrm{OpD}$ y la salud percibida, se puede observar también como numerosas de esas correlaciones negativas permanecen significativas, aun cuando controlamos el efecto de cada uno de los cinco factores de personalidad (Tabla 2), excepto cuando se controla el efecto de la variable Neuroticismo. Donde no se aprecia correlación significativa es en la puntuación total de salud y en las subescalas: Síntomas Somáticos, Ansiedad y Depresión. Cuando se controla el efecto de la variable Extraversión, no se aprecia una correlación significativa en las subescalas Síntomas
Somáticos y Ansiedad. Y por último, controlado el efecto de la variable Apertura, la correlación no es significativa para la subescala Síntomas Somáticos.

Tal y como podemos observar en la siguiente Tabla (3), existen de diferencias significativas entre los pesimistas y los optimistas respecto a la salud percibida; así, son los pesimistas quienes significativamente tienen mayores puntuaciones, indican tener peor Salud General, mayor Disfunción Social y mayores niveles de Depresión. Respecto a los Síntomas Somáticos y la Ansiedad, las diferencias entre pesimistas y optimistas no son estadísticamente significativas, pero son los primeros quienes presentan niveles superiores en ambas puntuaciones, por lo tanto, mayor nivel de Síntomas Somáticos y mayor Ansiedad.

En la Figura 1, se presenta el modelo de regresión (pasos sucesivos) de la influencia del $\mathrm{OpD}$ y los Cinco Grandes, sobre las cuatro subescalas del

\section{TABLA 3}

Estadísticos descriptivos y prueba t de Student para dos muestras independientes (optimistas y pesimistas)

\begin{tabular}{lcccccccccc}
\hline & \multicolumn{2}{c}{ GHQ TOTAL } & \multicolumn{2}{c}{ GHQ A } & \multicolumn{2}{c}{ GHQ B } & \multicolumn{2}{c}{ GHQ C } & \multicolumn{2}{c}{ GHQ D } \\
\hline & $\mathrm{M}$ & $\mathrm{DE}$ & $\mathrm{M}$ & $\mathrm{DE}$ & $\mathbf{M}$ & $\mathrm{DE}$ & $\mathrm{M}$ & $\mathrm{DE}$ & $\mathrm{M}$ & $\mathrm{DE}$ \\
\hline Pesimistas & 47.22 & 9.495 & 11.96 & 3.284 & 12.26 & 3.575 & 14.55 & 2.285 & 8.8 & 3.193 \\
Optimistas & 43.73 & 9.823 & 11.36 & 3.373 & 11.53 & 4.028 & 13.69 & 2.166 & 8.01 & 2.13 \\
$t_{(2,193)}$ & $2.506^{*}$ & \multicolumn{2}{c}{1.248} & \multicolumn{2}{c}{1.345} & $2.639 * *$ & $2.062 *$ \\
\hline
\end{tabular}

Nota. Se ha asumido la igualdad de varianzas salvo en la variable dependiente GHQ D, donde la prueba Levene $(p<0.05)$ hace no asumir la igualdad de las varianzas. GHQ A (Factor Síntomas Somáticos del Cuestionario de Salud General de Goldberg); GHQ B (Factor Ansiedad del Cuestionario de Salud General de Goldberg); GHQ C (Factor Disfunción Social del Cuestionario de Salud General de Goldberg); GHQ D (Factor Depresión del Cuestionario de Salud General de Goldberg).

$* p<0.05 . * * p<0.01$.

Fuente: elaboración propia 
Cuestionario de Salud General de Goldberg. Así, las puntuaciones totales de percepción de salud vienen explicadas en un 60\% por el factor Depresión, en un $24 \%$ por los Síntomas Somáticos, y en un $7 \%$ y $4 \%$, por el factor Ansiedad y Disfunción Social, respectivamente. En el factor Depresión influyen solo dos factores: Neuroticismo y Amabilidad. El primero, Neuroticismo, está influenciado por la Extraversión, el OpD, la Apertura y la Responsabilidad. Mientras que solo el primero y el último, la Extraversión y la Responsabilidad, influyen en la Amabilidad. Respecto a los Síntomas Somáticos, solo tiene influencia el factor Neuroticismo, explicando el 19\% de la varianza en las puntuaciones. La varianza de la subescala Ansiedad viene explicada por los factores Neuroticismo y Responsabilidad, en un $22 \%$ y $3 \%$, respectivamente. El factor Responsabilidad está influido por el factor Amabilidad, Apertura y Neuroticismo. La última de las subescalas del GHQ-28, Disfunción Social, viene explicada por el factor Neuroticismo, el OpD y la Responsabilidad, en un $11 \%, 5 \%$ y $4 \%$, respectivamente. Finalmente destacar que las puntuaciones de $\mathrm{OpD}$ también han sido analizadas mediante el modelo de regresión, mostrándose explicada su varianza un $13 \%$ por el factor Apertura, un 6\% por la subescala del GHQ28 Disfunción Social, siendo por tanto esta relación bidireccional, un 3\% por el factor Amabilidad y, finalmente, también influye escasamente el factor Neuroticismo en un 2\%.

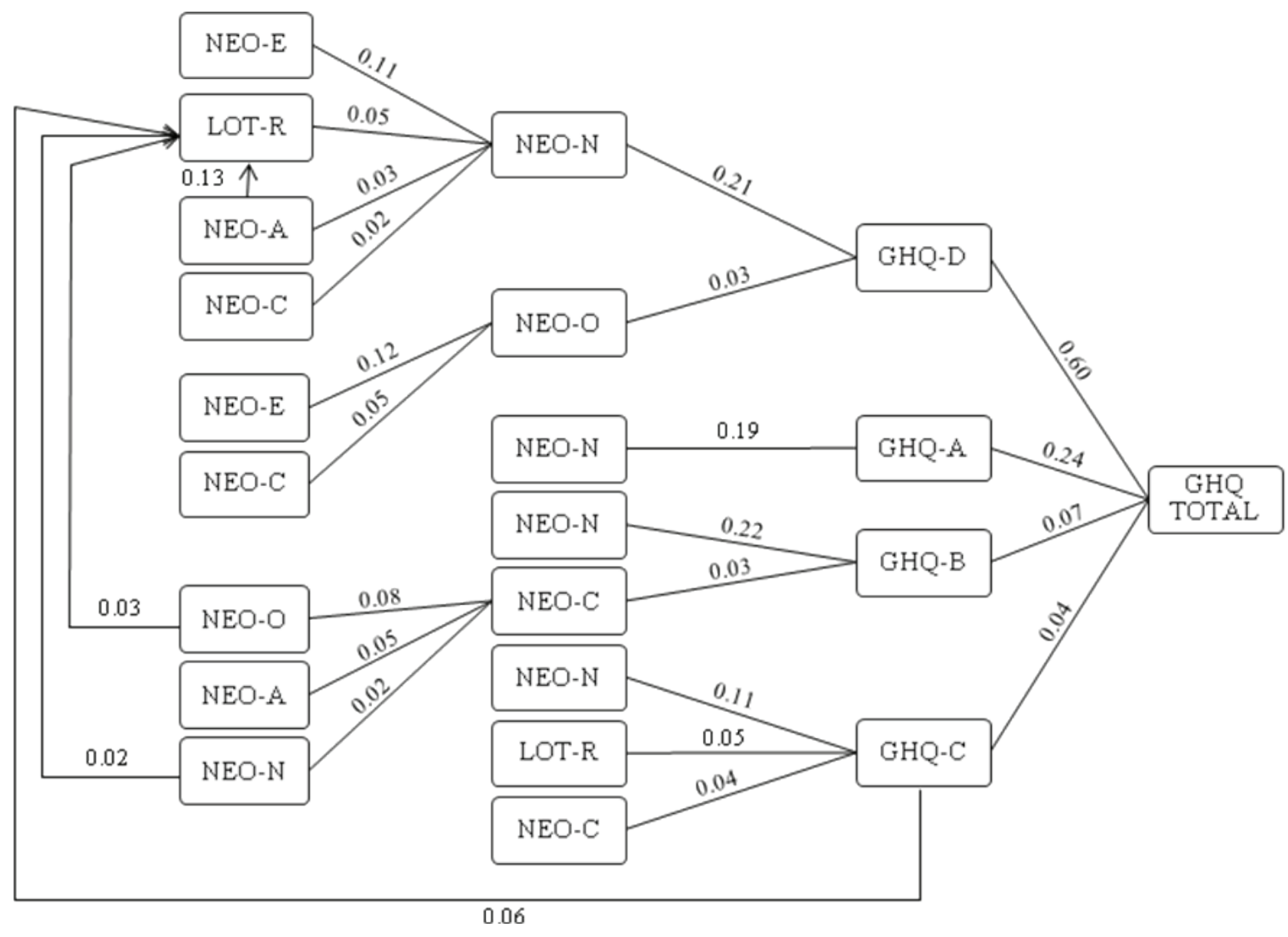

Figura 1. Modelo de análisis de regresión (método pasos sucesivos).

Nota. LOT-R (Puntuación Total del Test de Orientación Vital-Revisado); NEO N (Factor Neuroticismo); NEO E (Factor Extraversión); NEO O (Factor Amabilidad); NEO A (Factor Apertura); NEO C (Factor Responsabilidad); GHQ TOTAL (Puntuación Total del Cuestionario de Salud General de Goldberg ); GHQ A (Factor Síntomas Somáticos del Cuestionario de Salud General de Goldberg); GHQ B (Factor Ansiedad del Cuestionario de Salud General de Goldberg); GHQ C (Factor Disfunción Social del Cuestionario de Salud General de Goldberg); GHQ D (Factor Depresión del Cuestionario de Salud General de Goldberg).

Fuente: elaboración propia 


\section{Discusión}

El OpD está relacionado con la percepción de la salud y los Cinco Grandes factores de la personalidad (Carve et al., 2010). Esta relación es negativa respecto a la percepción de la salud, y es significativa entre ambas variables aun cuando se controla la influencia de los factores de personalidad analizados, manteniéndose sobre todo para la subescala Disfunción Social, Depresión y puntuación total del GHQ-28, aunque en estos dos últimos casos, deja de ser significativa cuando se controla la influencia del factor de personalidad, Neuroticismo. Ser optimista o pesimista lleva asociado una percepción diferente de la propia salud: los pesimistas muestran peor percepción general de su salud (Scheier \& Carver, 1985), mayor Disfunción Social y mayores niveles de Depresión (Giltay et al., 2006).

Sin embargo, respecto a los factores de personalidad analizados, dicha correlación es positiva excepto en el factor Neuroticismo. Respecto a este último, son diversos los estudios que aprecian índices de correlación superiores a los encontrados en el presente trabajo (Chico, 2002; Darvill \& Johnson, 1991; Scheier et al., 1994), quizás, debido a que analizan etapas evolutivas diferentes, estando la vejez marcada por niveles menores de Neuroticismo (Costa \& McCrae, 1988). No obstante, se encuentra presente en la explicación de alrededor del 20\% de la varianza de las subescalas de salud analizadas, salvo la de Disfunción Social que supera escasamente el 10\%, y a su vez, está influenciado por la Extraversión, el OpD, la Apertura y la Responsabilidad.

Por lo tanto, el factor Neuroticismo está presente en todas las escalas de percepción de salud analizadas, siendo el único aspecto de la personalidad que influye en los Síntomas Somáticos, y debiendo tener presente que este factor puede estar contaminando la respuesta de los sujetos (Rasmussen et al., 2009), ya que se evalúa mediante la realización de autoinformes, y se ha comprobado que el Neuroticismo está asociado con la percepción de síntomas injustificados (Feldman et al., 1999). El análisis de la subescala de Depresión nos muestra que, además del Neuroticismo, está relacionada con el factor Amabilidad; en la subescala Ansiedad, al
Neuroticismo se añade el factor Responsabilidad y, por último, en la subescala Disfunción social, influyen además el Optimismo vital y la Responsabilidad. Por tanto, salvo en la Disfunción Social, con la que posee una relación bidireccional, el $\mathrm{OpD}$ no influye directamente sobre la salud, sino que lo hace de modo indirecto mediando en el factor Neuroticismo, e influyendo en el propio $\mathrm{OpD}$ en otros factores de la personalidad, como Apertura, Amabilidad y Neuroticismo.

En la percepción de la salud existen diferencias significativas entre los optimistas y los pesimistas, lo que nos lleva a pensar que tiene una mayor importancia el Optimismo vital y que su influencia puede ser indirecta a través de otros factores no analizados en el presente estudio, como el ambiente familiar (Heinonen et al., 2005), la realización de actividades sociales y educativas, etc. También podrían ser analizadas variables más objetivas, intentando mitigar así la posible contaminación que previamente se ha señalado del Neuroticismo sobre la percepción del estado de salud (Rasmussen et al., 2009), como el estatus socioeconómico (Heinonen et al., 2006), la edad, el género, la discapacidad física (Lee \& Shinkai, 2003) o la capacidad para la realización de actividades físicas y sociales (Leinonen, Heikkinen \& Jylhä, 2002), con el objetivo de descubrir todas las variables que influyen en el nivel de $\mathrm{OpD}$ de la persona.

\section{Referencias}

Atienza, A. A., Stephens, M. A. P. \& Townsend, A. L. (2004). Role stressors as predictors of changes in women's optimistic expectations. Personality and Individual Differences, 37(3), 471-484.

Booth-Kewley, S. \& Friedman, H. S. (1987). Psychological predictors of heart disease: A quantitative review. Psychological Bulletin, 101(3), 343-362.

Cabanach, R., Fariña, F., Freire, C., González, P. \& Ferradás, Mํ.Mํ. (2013). Diferencias en el afrontamiento del estrés en estudiantes universitarios hombres y mujeres. European Journal of Education and Psychology, 6(1), 19-32.

Cacho, L. J., García-García, R., Arcaya J., Vicente, J. L. \& Lantada, N. (1999). Una propuesta de aplicación 
y puntuación del test del reloj en la enfermedad de Alzheimer. Revista de Neurología, 28(7), 648-655.

Carver, C. S., Scheier, M. F. \& Segerstrom, S. C. (2010). Optimism. Clinical Psychology Review, 30(7), 879889.

Charles, S. T., Reynolds, C. A. \& Gatz, M. (2001). Agerelated differences and change in positive and negative affect over 23 years. Journal of Personality and Social Psychology, 80(1), 136-151.

Chico, E. (2002). Optimismo disposicional como predictor de estrategias de afrontamiento. Psicothema, 14(3), 544-550.

Costa, P. T. \& McCrae, R. R. (1985). Hypochondriasis, neuroticism, and aging: When are somatic complaints unfounded? American Psychologist, 40(1), 19-28.

Costa, P. T. \& McCrae, R. R. (1988). Personality in adulthood: A six-year longitudinal study of selfreports and spouse ratings on the NEO-PI. Journal of Personality and Social Psychology, 54(5), 853-863.

Costa, P. T. \& McCrae, R. R. (1999). Inventario de Personalidad NEO revisado (NEO PI-R) e Inventario NEO reducido de Cinco Factores (NEOFFI). Manual profesional. Madrid: TEA.

Darvill, T. J. \& Johnson, R. C. (1991). Optimism and perceived control of life events as related to personality. Personality and Individual Differences, 12(9), 951-954.

De la Cruz-Sánchez, E., Feu, S. \& Vizuete-Carrizosa, M. (2013). El nivel educativo como factor asociado al bienestar subjetivo y la salud mental en la población española. Universitas Psychologica, 12(1), 31-40. Feldman, P., Cohen, S., Doyle, W. J., Skoner, D. P. \& Gwaltney, J. M. (1999). The impact of personality on the reporting of unfounded symptoms and illness. Journal of Personality and Social Psychology, 77(2), 370-378.

Del Barrio, C., Barrios, A., Granizo, L., van der Meulen, K., Andrés, S. \& Gutiérrez, H. (2011). Contribuyendo al bienestar emocional de los compañeros: evaluación del Programa Compañeros Ayudantes en un instituto madrileño. European Journal of Education and Psychology, 4(1), 5-17.

Friedmand, M. \& Rosenman, R. H. (1959). Association of a specific overt behavior pattern with increases in blood cholesterol, blood clotting time, incidence of Arcus Senilis and Clinical Coronary Artery Disease. Journal of the American Medical Association, 169(12), 1286-1296.

Gázquez, J. J., Pérez, M. C. \& Carrión, J. J. (2010). Análisis de la Memoria Cotidiana en alumnos del Programa Universitario para Mayores en Almería. European Journal of Education and Psychology, 3(1), 155-165.

Giltay, E. J., Zitman, F. G. \& Kromhout, D. (2006). Dispositional optimism and the risk of depressive symptoms during 15 years of follow-up: The Zutphen Elderly Study. Journal of Affective Disorders, 91(1), 45-52.

Goldberg, D. P. \& Hillier, V. F. (1979). A scaled version of the General Health Questionnaire. Psychological Medicine, 9(1), 139-145.

Gómez-Angulo, C. B. \& Campo-Arias, A. (2011). Escala de Yesavage para Depresión Geriátrica (GDS-15 y GDS-5): estudio de la consistencia interna y estructura factorial. Universitas Psychologica, 10(3), 735-743.

Heinonen, K., Räikkönen, K. \& Keltikangas-Järvinen, L. (2005). Dispositional optimism: Development over 21 years from the perspectives of perceived temperament and mothering. Personality and Individual Differences, 38(2), 425-435.

Heinonen, K., Räikkönen, K., Matthews, K. A., Scheier, M. F., Raitakari, O. T., Pulkki, L., et al. (2006). Socioeconomic status in childhood and adulthood: Associations with dispositional optimismand pessimismover a 21-year follow-up. Journal of Personality, 74(4), 1111-1126.

Inglés, C. J., Martínez-González, A.E. \& García-Fernández, J. M. (2013). Conducta prosocial y estrategias de aprendizaje en una muestra de estudiantes españoles de Educación Secundaria Obligatoria. European Journal of Education and Psychology, 6(1), 33-53.

Ingledew, D. K. \& Brunning, S. (1999). Personality, preventive health behavior and comparative optimism about health problems. Journal of Health Psychology, 4(2), 193-208.

Johnson, S. J., Batey, M. \& Holdsworth, L. (2009). Personality and health: The mediating role of Trait Emotional Intelligence and Work Locus of 


\section{REPERCUSión DEL OPTIMISMO Y DE LOS CINCO GRANDES FACTORES DE LA PERSONALIDAD SOBRE LA SALUD DE PERSONAS MAYORES}

Control. Personality and Individual Differences, 47(5), 470-475.

Lauriola, M., Russo, P. M., Lucidi, F., Violani, C. \& Levin, I. P. (2005). The role of personality in positively and negatively framed risky health decisions. Personality and Individual Differences, 38(1), 45-59.

Lee, Y. \& Shinkai, S. (2003). A comparison of correlates of self-rated health and functional disability of older persons in the Far East: Japan and Korea. Archives of Gerontology and Geriatrics, 37(1), 63-76.

Leinonen, R., Heikkinen, E. \& Jylhä, M. (2002). Changes in health, functional performance and activity predict changes in self-rated health: A 10-year follow-up study in older people. Archives of Gerontology and Geriatrics, 35(1), 79-92.

Londoño, C., Hernández, L. M., Alejo, I. E. \& Pulido, D. (2013). Diseño y validación de la Escala de Optimismo Disposicional/Pesimismo-EOP. Universitas Psychologica, 12(1), 139-155

Márquez, M., Fernández, M. I., Montorio, I. \& Losada, A. (2008). Experiencia y regulación emocional a lo largo de la etapa adulta del ciclo vital: análisis comparativo en tres grupos de edad. Psicothema, 20(4), 616-622.

Martínez, A., Reyes, G. A., García, A. \& González, M. I. (2006). Optimismo/pesimismo disposicional y estrategias de afrontamiento del estrés. Psicothema, 18(1), 66-72.

Meliá, J. L. \& Becerril, M. (2009). Health behaviour and safety in the construction sector. Psicothema, 21(3), 427-432.

Otero, J. M., Luengo, A., Romero, E., Gómez, J. A. \& Castro, C. (1998). Psicología de la personalidad. Manual de prácticas. Barcelona: Ariel Practicum.

Oyanadel, C. R. \& Buela-Casal, G. (2011). La percepción del tiempo: influencias en la salud física y mental. Universitas Psychologica, 11(1), 149-162.

Plomin, R., Scheier, M. F., Bergeman, C. S., Pedersen, N. L., Nesselroade, J. R. \& McClearn, G. E. (1992). Optimism, pessimism, and mental health: A twin/ adoption analysis. Personality and Individual Differences, 13(8), 921-930.

Radcliffe, N. M. \& Klein, W. M. P. (2002). Dispositional, unrealistic, and comparative optimism: Differential relations with the knowledge and processing of risk information and beliefs about personal risk.
Personality and Social Psychology Bulletin, 28(6), 836-846.

Rasmussen, H. N., Scheier, M. F. \& Greenhouse, J. B. (2009). Optimism and physical health: A metaanalytic review. Annals of Behavioral Medicine, 37(3), 239-256.

Rieffe, C., Villanueva, L., Adrián, J. E. \& Górriz, A. B. (2009). Quejas somáticas, estados de ánimo y conciencia emocional en adolescentes. Psicothema, 21(3), 459-464.

Rodríguez, C., González-Castro, P., Vicente, L., Núñez, J. C., González-Pienda, J. A, Álvarez, D., et al. (2011). Nuevas técnicas de evaluación en el Trastorno por Déficit de Atención e Hiperactividad (TDAH). European Journal of Education and Psychology, 4(1), 63-73.

Scheier, M. F. \& Carver, C. S. (1985). Optimism, coping and health: Assessment and implications of generalized outcome expectancies. Health Psychology, 4(3), 219-247.

Scheier, M. F. \& Carver, C. S. (1987). Dispositional optimism and physical well-being: The influence of generalized outcome expectancies on health. Journal of Personality, 55(2), 169-210.

Scheier, M., Carver, C. \& Bridges, M. (1996). Distinguishing optimism from neuroticism (and trait anxiety, self-mastery, and self-esteem): A reevaluation of the Life Orientation Test. Journal of Personality and Social Psychology, 67(6), 1063-1078.

Segerstrom, S. C. (2007). Optimism and resources: Effects on each other and on health over 10 years. Journal of Research in Personality, 41(4), 772-786.

Shnek, Z. M., Irvine, J., Stewart, D. \& Abbey, S. (2001). Psychological factors and depressive symptoms in ischemic heart disease. Health Psychology, 20(2), 141-145.

Sweeny, K., Carroll, P. J. \& Shepperd, J. A. (2006). Is optimism always best? Current Directions in Psychological Science, 15(6), 302-306.

Torregrosa, M. S., Inglés, C. J., García-Fernández, J. M., Valle, A. \& Núñez, J. C. (2012). Relaciones entre conducta agresiva y metas académicas: estudio con una muestra de estudiantes españoles de Educación Secundaria Obligatoria. Universitas Psychologica, 11(4), 1303-1315. 
Vizcarra, M. B. \& Pòo, A. M. (2011). Violencia de pareja en estudiantes universitarios del sur de Chile . Universitas Psychologica, 11(1), 89-98.

Vollrath, M. E. \& Torgersen, S. (2008). Personality types and risky health behaviors in Norwegian students. Scandinavian Journal of Psychology, 49(3), 287-292.
Zanon, C. \& Hutz, C. S. (2013). Affective Disposition, Thinking Styles, Neuroticism and Life Satisfaction. Universitas Psychologica, 12(2), 403-411. 